Revista Integración, temas de matemáticas

Escuela de Matemáticas

Universidad Industrial de Santander

Vol. 38, $\mathrm{N}^{\circ} 1,2020$, pág. 67-79

DOI: http://dx.doi.org/10.18273/revint.v38n1-2020006

\title{
The Dixmier trace and the Wodzicki residue for global pseudo-differential operators on compact manifolds
}

\author{
Dután CARdona $^{a *}$, CÉsar Del Corral ${ }^{b}$ \\ ${ }^{a}$ Ghent University, Department of Mathematics: Analysis, Logic and Discrete \\ Mathematics, Ghent, Belgium. \\ ${ }^{b}$ Universidad de los Andes, Department of Mathematics, Bogotá, Colombia.
}

\begin{abstract}
In this note, we announce the results of our investigation on the Dixmier trace and the Wodzicki residue for pseudo-differential operators on compact manifolds. We give formulae for the Dixmier trace and the non-commutative residue (also called Wodzicki's residue) of invariant pseudo-differential operators on compact manifolds with or without boundary. For every closed manifold, the notion of global symbol for invariant pseudo-differential operators will be based on the Fourier analysis associated to every elliptic and positive operator (developed by M. Ruzhansky, V. Turunen and J. Delgado). In particular, for each compact Lie group we will use its representation theory. For the analysis of operators on compact manifolds with boundary, we will use the non-harmonic analysis associated with boundary valued problems (developed by M. Ruzhansky, N. Tokmagambetov, and J. Delgado).
\end{abstract}

Keywords: Dixmier trace, non commutative residue, global operators, representation theory.

MSC2010: 81Q10, 47B10.

\section{La traza de Dixmier y el residuo de Wodzicki para operadores pseudodiferenciales globales sobre variedades compactas}

Resumen. En esta nota se anuncian los resultados de nuestra investigación sobre la traza de Dixmier y el residuo de Wodzicki para operadores pseudodiferenciales sobre variedades compactas. Se calcula la traza de Dixmier y el residuo no conmutativo (residuo de Wodzicki) de operadores pseudodiferenciales invariantes sobre variedades compactas con o sin borde. Para

\footnotetext{
*E-mail: duvanc306@gmail.com

Received: 17 July 2019, Accepted: 16 January 2020.

To cite this article: D. Cardona and C. del Corral, The Dixmier trace and the Wodzicki residue for global pseudo-differential operators on compact manifolds, Rev. Integr. temas mat. 38 (2020), No. 1, 67-79.
} 
cada variedad cerrada (suave, compacta y sin borde), se emplea la noción de símbolo global que viene dada por el análisis de Fourier asociado a cada operador elíptico y positivo (desarrollado por M. Ruzhansky and V. Turunen para para grupos de Lie y por M. Ruzhansky, N. Tokmagambetov y J. Delgado para variedades cerradas). En particular, para cada grupo de Lie compacto, se usa su teoría de representación. Respecto al análisis de operadores sobre variedades con borde, se usa el análisis no armónico asociado a problemas con valores de frontera (introducido por M. Ruzhansky, N. Tokmagambetov, y J. Delgado).

Palabras clave: Traza de Dixmier, residuo no conmutativo, operador global, teoría de representaciones.

\section{Introduction}

In this work, we classify the Dixmier traceability of some invariant operators on compact manifolds and we apply these results in order to study the Wodzicki residue of pseudodifferential operators. This work is a subsequent analysis to the program of M. Ruzhansky and J. Delgado on the classification of global operators in ideals contained in the algebra of bounded operators on Lebesgue spaces (see [16, 17, 18, 19, 20, 21]). The topic has been of intense research in the last years not only for operators on compact manifolds, but also on non-compact manifolds and other spaces without a differentiable structure (see e.g. $[1,3,4,5,6,8,9,10,11,14,15,27,24]$ ). The novelty of the announced results is that we use the matrix-valued global quantisation developed by Ruzhansky, Turunen, Tokmagambetov and Delgado, instead of the classical results on the subject based on the classical notion of the local symbol via localizations (see, e.g. Hörmander [26]), as in $[13,23,25,33,34]$ and references therein. We summarise our investigation in the following results:

- We provide necessary and sufficient conditions in order that global invariant pseudodifferential operators on a manifold with or without boundary belong to the Dixmier class $\mathcal{L}^{(1, \infty)}\left(L^{2}\right)$.

- We provide sufficient and necessary conditions in order to obtain Dixmier traceability for a type of pseudo-differential operators with global symbols in the Hörmander classes on compact Lie groups, and we express our results by using the representation theory of these groups. Also, we use Connes' trace theorem in order to get formulae for the noncommutative residue of classical pseudo-differential operators on compact Lie groups.

- For a smooth compact manifold with or without boundary, we find criteria in terms of the global symbols in order that the corresponding operators belong to the Marcinkiewicz ideal $\mathcal{L}^{(p, \infty)}\left(L^{2}\right), 1<p<\infty$, as well as to the Dixmier ideal $\mathcal{L}^{(1, \infty)}(H)$ where $H=L^{2}(M)$.

Our main result will be presented in Section 3. In Section 2 we provide some basics on the theory of pseudo-differential operators, the Wodzicki residue and the Dixmier trace. Finally, in Section 4 we provide some examples.

[Revista Integración, temas de matemáticas 


\section{Preliminaries}

In order to present our main results we recall some definitions (see Connes [13]). Let $H$ be a Hilbert space, $\mathcal{L}(H)$ be the algebra of bounded linear operators on $H$ and $\mathcal{K}(H)$ be the ideal of compact operators on $H$. A compact operator $A \in \mathcal{K}(H)$ belongs to the Dixmier class $\mathcal{L}^{(1, \infty)}(H)$ if

$$
\sum_{1 \leq n \leq N} s_{n}(A)=O(\log (N)), \quad \text { as } N \rightarrow \infty,
$$

where $\left\{s_{n}(A)\right\}$ denotes the sequence of singular values of $A$, which consists of the points in the spectrum of $\sqrt{A^{*} A}$. The sequence $s_{n}(A)$ can be arranged in increasing order so that $\mathcal{L}^{(1, \infty)}(H)$ may be endowed with the norm

$$
\|A\|_{\mathcal{L}^{(1, \infty)}(H)}=\frac{1}{\log N} \sup _{N>1} \sum_{n \leq N} s_{n}(A) .
$$

If $A \in \mathcal{L}^{(1, \infty)}(H)$, the functional $\|A\|_{\mathcal{L}^{(1, \infty)}}(H)$ defines a norm. Some ideals, closely related to the Dixmier ideal, are Marcinkiewicz ideals $\mathcal{L}^{(p, \infty)}(H)$, defined by those bounded linear operators $A$ on $L^{2}$ satisfying the eigenvalue condition:

$$
\sum_{1 \leq n \leq N} s_{n}(A)=O\left(N^{(1-1 / p)}\right), N \rightarrow \infty,
$$

where $1<p<\infty$. On the class $\mathcal{L}^{(p, \infty)}\left(L^{2}\right)$ the usual norm is given by

$$
\|A\|_{\mathcal{L}^{(p, \infty)}(H)}:=\sup _{N \geq 1} N^{\left(\frac{1}{p}\right)-1} \sum_{1 \leq n \leq N} s_{n}(A) .
$$

The main objects here are pseudo-differential operators, which we introduce as follows $([16,26,31])$. A pseudo-differential operator $A$ is an operator defined by the integral representation

$$
A f(x)=\int_{\mathbb{R}^{n}} e^{i x \cdot \xi} \sigma^{A}(x, \xi) \hat{f}(\xi) d \xi,
$$

where the function $\sigma^{A}(x, \xi)$-called the symbol of $A$ - satisfies estimates of the type (see Hörmander [26])

$$
\left|\partial_{x}^{\alpha} \partial_{\xi}^{\beta} \sigma^{A}(x, \xi)\right| \leq C_{\alpha, \beta}(1+|\xi|)^{m-|\beta|} .
$$

Here $\hat{f}$ denotes the euclidean Fourier transform of the function $f$ and $m$ is the order of the pseudo-differential operator $A$. If we consider a compact manifold without boundary $M$ of dimension $\varkappa$, a pseudo-differential operator $A$ on $M$ can be defined by using the notion of local symbol; this means that for any local chart $U$, the operator $A$ has the form

$$
A u(x)=\int_{T_{x}^{*} U} e^{i x \cdot \xi} \sigma^{A}(x, \xi) \widehat{u}(\xi) d \xi .
$$

A pseudo-differential operator $A$ is called classical, if $\sigma^{A}$ admits an asymptotic expansion $\sigma^{A}(x, \xi) \sim \sum_{j=0}^{\infty} \sigma_{m-j}^{A}(x, \xi)$ in such a way that each function $\sigma_{m-j}(x, \xi)$ is homogeneous 
in $\xi$ of order $m-j$ for $\xi \neq 0$. The set of classical pseudo-differential operators of order $m$ is denoted by $\Psi_{c l}^{m}(M)$. For $A \in \Psi_{c l}^{m}(M)$, and for $x \in M$,

$$
\operatorname{res}_{x}(A)=\int_{|\xi|=1} \sigma_{-\varkappa}(x, \xi) d \xi
$$

defines a local density which can be glued over $M$. In this case, the non-commutative residue of $A$ is defined by the expression

$$
\operatorname{res}(A)=\frac{1}{\varkappa(2 \pi)^{\varkappa}} \int_{M} \int_{|\xi|=1} \sigma_{-\varkappa}^{A}(x, \xi) d \xi d x .
$$

The following fact is due to Connes, [13]: if $A \in \Psi_{c l}^{-\varkappa}(M)$ is a positive operator, then $\operatorname{Tr}_{\omega}(A)=\operatorname{res}(A)$.

In the case of a manifold $M$ with boundary, the typical algebra of pseudo-differential operators is known as the Boutet de Monvel algebra (BdM). This algebra is formed by matrix valued pseudo-differential operators associated with boundary value problems (see [2]):

$$
A=\left[\begin{array}{cc}
P_{+}+G & K \\
T & S
\end{array}\right]: C^{\infty}(M) \oplus C^{\infty}(\partial M) \rightarrow C^{\infty}(M) \oplus C^{\infty}(\partial M) .
$$

The noncommutative residue and the Dixmier trace for an operator $P$ in BdM's algebra have been studied e.g. in Fedosov [23] and Nest and Schrohe [28].

In order to study the Dixmier trace and the non-commutative residue, we will use the notion of global pseudo-differential operator. If we consider a closed manifold $M$ (i.e., a compact manifold without boundary) and $H=L^{2}(M)$, there exists a global Fourier analysis associated to every positive elliptic pseudo-differential operator $E$ on $M$ which gives for certain pseudo-differential operators $A,-$ called $E$-invariants-, a discrete Fourier representation of the form

$$
A f(x)=\sum_{l=0}^{\infty}\left\langle\sigma_{A, E}(l) \widehat{f}(l), e_{l}(x)\right\rangle_{\mathbb{C}^{d_{l}}},
$$

where $e_{l}(x):=\left(e_{l}^{m}\right)_{1 \leq m \leq d_{l}}$ and $\left\{e_{l}^{m}: l \in \mathbb{N}, 1 \leq m \leq d_{l}\right\}$ is a basis of $L^{2}(M)$ consisting of eigenfunctions $\lambda_{l}, l \in \mathbb{N}_{0}$, of $E$. The function $\sigma_{A, E}$ is called the matrix valued symbol of $A$ with respect to $E$. The quantisation procedure associating to every operator $A$ acting in $C^{\infty}(M)$ the matrix valued symbol $\sigma_{A, E}$ was introduced by J. Delgado and M. Ruzhansky, [20]. Also, If $M=G$ is a compact Lie group and $E=-\mathcal{L}_{G}$ is the positive Laplace-Beltrami operator on $G$, Ruzhansky and Turunen in [31] give a discrete representation to every pseudo-differential operator $A$ acting on $C^{\infty}(G)$ in terms of the representation theory of the group $G$, in the following way:

$$
A f(x)=\sum_{[\xi] \in \widehat{G}} d_{\xi} \operatorname{Tr}\left[\xi(x) \sigma_{A}(x, \xi) \widehat{f}(\xi)\right] .
$$

Here $\widehat{G}$ denotes the unitary dual of $G$. Ruzhansky-Turunen's calculus that gives a characterisation for the Hörmander classes $\Psi_{\rho, \delta}^{m}(G)$ on a compact Lie group $G$ by using global 
symbols. In fact, $A \in \Psi_{\rho, \delta}^{m}(G)$, if and only if, its global symbol $\sigma_{A}(x, \xi)$ as in (5) satisfies $\left\|\Delta_{\xi}^{\alpha} \partial_{x}^{\beta} \sigma_{A}(x, \xi)\right\|_{o p} \leq C\langle\xi\rangle^{m-\rho|\alpha|+\delta|\beta|}, \quad x \in G,[\xi] \in \widehat{G}$, where $\langle\xi\rangle:=\left(1+\lambda_{[\xi]}\right)^{\frac{1}{2}}$ and $\left\{\lambda_{[\xi]}\right\}$ is the spectrum of $-\mathcal{L}_{G}$, which can be enumerated by $[\xi] \in \widehat{G}$.

Let us observe that if $M=G$ is a compact Lie group and the operator $E \in \Psi_{+e}^{\nu}(G)$ is not invariant (this means that the global symbol $\sigma_{E}(x, \xi)$ depends of both variables, the spatial variable $x \in G$, and the Fourier variables $[\xi] \in \widehat{G})$, then in local coordinates, $E$-invariants operators $A$ are $E$-multipliers, but they are not Fourier multipliers because in local coordinates their symbols are depending on the spatial variables.

In the formulation of our result for compact manifolds with boundary, we use the global quantization of pseudo-differential operators on compact manifolds with boundary due to J. Delgado, M. Ruzhansky and N. Tokmagambetov [30] which we briefly describe as follows. Let $M$ be a compact manifold with boundary $\partial M$ and let $L$ be a pseudodifferential operator on $M$ satisfying some boundary conditions on $\partial M$. We assume that $L$ has discrete spectrum $\left\{\lambda_{\xi} \mid \xi \in \mathcal{I}\right\}$. If $\sigma_{A, L}: M \times \mathcal{I} \rightarrow \mathbb{C}$ is a suitable function, the operator $A$ associated with $\sigma_{A}$ can be written as

$$
A f(x)=\sum_{\xi \in \mathcal{I}} u_{\xi}(x) \sigma_{A, L}(x, \xi) \widehat{f}(\xi), \text { for } f \in C_{L}^{\infty}(M),
$$

where $\widehat{f}:=\mathcal{F}_{L}(f)$ denotes the $L$-Fourier transform of $f$ introduced in [30]. $L$-Fourier multipliers are bounded linear operators $A: C_{L}^{\infty}(M) \rightarrow C_{L}^{\infty}(M)$ satisfying $\mathcal{F}_{L}(A f)(\xi)=$ $\sigma_{A, L}(\xi) \mathcal{F}_{L}(f)(\xi)$, for all $f \in C_{L}^{\infty}(M)$, and for some function $\sigma_{A, L}: \mathcal{I} \rightarrow \mathbb{C}$ depending only on the Fourier variables $\xi \in \mathcal{I}$. In this case, $\sigma_{A, L}(\xi)$ is called the $L$-symbol of $A$.

\section{The Dixmier Trace and the non-commutative residue for global pseudo-differential operators}

In this section, we present our main results for global operators on compact manifolds. We start with the case of compact manifolds without boundary. Here, $\varkappa$ denotes the dimension of a fixed closed manifold.

Theorem 3.1. Let $M$ be a $\varkappa$-dimensional compact manifold without boundary and let $E \in \Psi_{+e}^{\nu}(M)$ be a positive elliptic pseudo-differential operator on $M$. If $A: L^{2}(M) \rightarrow$ $L^{2}(M)$ is an E-invariant bounded operator with matrix-valued symbol $\left(\sigma_{A, E}(l)\right)_{l}$, then we have that

- $A$ is Dixmier traceable, i.e., $A \in \mathcal{L}^{(1, \infty)}\left(L^{2}(M)\right)$ if, and only if,

$$
\tau(A):=\frac{1}{\operatorname{dim}(M)} \lim _{N \rightarrow \infty} \frac{1}{\log N} \sum_{l:\left(1+\lambda_{l}\right)^{\frac{1}{\nu}} \leq N} \operatorname{Tr}\left(\left|\sigma_{A, E}(l)\right|\right)<\infty,
$$

where $\sigma_{A, E}$ is as in (4). Moreover, if $A$ is positive, $\tau(A)=\operatorname{Tr}_{w}(A)$.

- If $A$ is an E-invariant operator, then $A \in \mathcal{L}^{(p, \infty)}\left(L^{2}(M)\right)$ if, and only if,

$$
\gamma_{p}(A):=\sup _{N \geq 1} N^{\operatorname{dim} M\left(\frac{1}{p}-1\right)} \cdot \sum_{l:\left(1+\lambda_{l}\right)^{\frac{1}{\nu}} \leq N} \operatorname{Tr}\left(\left|\sigma_{A, E}(l)\right|\right)<\infty,
$$

where $1<p<\infty$. In this case, $\|A\|_{\mathcal{L}^{(p, \infty)}\left(L^{2}(M)\right)} \asymp \gamma_{p}(A)$. 
- Let $M=G$ be a compact Lie group and let $\widehat{G}$ be the unitary dual of $G$. If we denote by $\sigma_{A}(x, \xi)$ the matrix valued symbol associated to $A$, then under the condition

$$
\left\|\Delta_{\xi}^{\alpha} \partial_{x}^{\beta} \sigma_{A}(x, \xi)\right\|_{o p} \leq C\langle\xi\rangle^{-\varkappa-|\alpha|}, x \in G,[\xi] \in \widehat{G}, \forall \alpha \in \mathbb{R}^{n},
$$

the operator $A$ is Dixmier measurable.

- If $A \in \Psi_{c l}^{-\varkappa}(G)$ is a classical and positive pseudo-differential operator and its symbol admits an asymptotic expansion in homogeneous components of the form:

$$
\sigma^{A}(x, \xi) \sim \sum_{j=0}^{\infty} a_{m-j}(x) \sigma^{A_{m-j}}(\xi)
$$

then

$$
\operatorname{res}(A)=\frac{1}{\operatorname{dim}(G)} \int_{G} a_{-\varkappa}(x) d x \times \lim _{N \rightarrow \infty} \frac{1}{\log N} \sum_{\xi:\langle\xi\rangle \leq N} d_{\xi} \operatorname{Tr}\left(\left|\sigma_{A_{-\varkappa}}(\xi)\right|\right) .
$$

The expression (11) in the preceding theorem gives a formula for the noncommutative residue of classical operators in terms of irreducible representations on $G$. We observe that our approach provide formulae for the noncommutative residue of operators on the torus, of a different way in relation with the recent work of Pietsch [29].

Now we present our result concerning to global operators on a manifold with boundary. With the notations above our result on the Dixmier traceability of operators on manifolds with boundary can be enunciated of the following way. We write $\mathcal{I}=\left\{\xi_{l}: l \in \mathbb{N}_{0}\right\}$.

Theorem 3.2. Let $M$ be a compact manifold with boundary $\partial M$. If $A: L^{2}(M) \rightarrow L^{2}(M)$ is a bounded Fourier multiplier, and $L$ is a self-adjoint operator on $L^{2}(M)$, then we have the following assertions:

- A is Dixmier traceable if, and only if,

$$
\tau^{\prime}(A):=\lim _{N \rightarrow \infty} \frac{1}{\log N} \sum_{l \leq N}\left|\sigma_{A, L}\left(\xi_{l}\right)\right|<\infty .
$$

In this case, for $A$ a positive operator, $\tau^{\prime}(A)=\operatorname{Tr}_{\omega}(A)$.

- Moreover, if $L$ is an operator of order $m$ satisfying the Weyl eigenvalue counting formula, that is,

$$
N_{L}(\lambda):=\#\left\{l:\left|\lambda_{\xi_{l}}\right|^{\frac{1}{m}} \leq \lambda\right\}=C_{0} \lambda^{\operatorname{dim} M}+O\left(\lambda^{\operatorname{dim} M-1}\right), \lambda \rightarrow \infty,
$$

then $A$ is Dixmier traceable if, only if (cf. (12)),

$$
\tau^{\prime}(A)=\frac{1}{\operatorname{dim} M} \lim _{N \rightarrow \infty} \frac{1}{\log N} \sum_{l:\left|\lambda_{\xi_{l}}\right|^{\frac{1}{m}} \leq N}\left|\sigma_{A, L}\left(\xi_{l}\right)\right|<\infty .
$$

In this case, for $A$ a positive operator, $\tau^{\prime}(A)=\operatorname{Tr}_{\omega}(A)$. 
- $A \in \mathcal{L}^{(p, \infty)}\left(L^{2}(M)\right)$ if, and only if,

$$
\gamma_{p}^{\prime}(A):=\sup _{N \geq 1} N^{\left(\frac{1}{p}-1\right)} \sum_{l \leq N}\left|\sigma_{A, L}\left(\xi_{l}\right)\right|<\infty,
$$

where $1<p<\infty$. In this case, $\|A\|_{\mathcal{L}^{(p, \infty)}\left(L^{2}(M)\right)} \sim \gamma_{p}^{\prime}(A)$. Moreover, if L satisfies (13), we have

$$
\gamma_{p}^{\prime}(A) \asymp \sup _{N \geq 1} N^{\operatorname{dim} M\left(\frac{1}{p}-1\right) .} \sum_{l:\left|\lambda_{\xi_{l}}\right|^{\frac{1}{m}} \leq N}\left|\sigma_{A, L}\left(\xi_{l}\right)\right| .
$$

On the other hand, let $A=\left[P_{+}, T\right]^{t}$ be a column elliptic operator in the Boutet de Monvel algebra of order $n$. We assume the existence of $L$ satisfying as in RuzhanskyTokmagambetov's calculus. Any parametrix $R$ of $P_{T}$ is Dixmier traceable and $\operatorname{Tr}_{\omega}(R)=$ $\operatorname{res}(R)=\lim _{N \rightarrow \infty} \frac{1}{\ln N} \sum_{l<N}\left|\left(\sigma_{P, L}\left(\xi_{l}\right)\right)^{-1}\right|$, here $\left(\sigma_{P, L}\left(\xi_{l}\right)\right)_{l \in \mathbb{N}_{0}}$ denotes the global symbol of the operator $P$ with respect to $L$. This residue's formula is the same for all parametrix of $A, R$ and independent of the boundary condition and independent of the average function $\omega$. If in addition L satisfies the Weyl Eigenvalue Counting Formula, we also have

$$
\operatorname{Tr}_{w}(A)=\frac{1}{\operatorname{dim}(M)} \lim _{N \rightarrow \infty} \frac{1}{\log N} . \sum_{l:\left|\lambda_{\xi_{l}}\right|^{\frac{1}{m}} \leq N}\left|\sigma_{A, L}\left(\xi_{l}\right)\right| .
$$

The proofs of these theorems and the corresponding analysis developed in this global setting can be found in Cardona and Del Corral [12]. We refer the reader to Cardona, Del Corral and Kumar [7] where the Dixmier trace of discrete pseudo-differential operators has been investigated.

\section{Examples}

In this section we provide some examples in relation with our main results. First, we consider a Dixmier traceable Bessel potential associated to $L$. Also, we consider an example of a Dixmier traceable operator on the manifold with boundary $M=[0,1]$. Later, we consider the case of Bessel potential on $\mathrm{SU}(2) \cong \mathbb{S}^{3}$ and $\mathrm{SU}(3)$ respectively.

Example 4.1. Let $M$ be a compact manifold with boundary $\partial M$ and $L$ as in the preceding sections. Let us consider the model operator $L_{M}$ self adjoint on $L^{2}(M)$. If we assume that $-L$ is a positive operator of order $\nu$, and some positive real number $s_{1}$ satisfies

$$
\sum_{l \in \mathbb{N}_{0}}\left\langle\xi_{l}\right\rangle^{-s}=\infty, \sum_{l \in \mathbb{N}_{0}}\left\langle\xi_{l}\right\rangle^{-s^{\prime}}<\infty
$$

for all $0<s \leq s_{1}<s^{\prime}<\infty$, then with the notations above the operator $A:=(I-L)^{-\frac{s_{1}}{\nu}}$ is Dixmier traceable on $L^{2}(M)$. For the proof, we combine that the global symbol of $A$ is given by

$$
\sigma_{A, L}\left(\xi_{l}\right)=\left(1-\lambda_{\xi_{l}}\right)^{-\frac{s_{1}}{\nu}} \asymp\left\langle\xi_{l}\right\rangle^{-s_{1}} .
$$

As consequence of (18) we have that $\left\langle\xi_{l}\right\rangle^{-s_{1}}=O\left(l^{-1}\right), l \rightarrow \infty$, and

$$
\sum_{l \leq N}\left\langle\xi_{l}\right\rangle^{-s_{1}}=O(\log N), N \rightarrow \infty .
$$

Vol. 38, $\left.\mathrm{N}^{\circ} 1,2020\right]$ 
Hence, by Theorem 3.2 we obtain

$$
\operatorname{Tr}_{w}\left((I-L)^{-\frac{s_{1}}{\nu}}\right) \asymp \lim _{N \rightarrow \infty} \frac{1}{\log N} \sum_{l \leq N}\left\langle\xi_{l}\right\rangle^{-s_{1}}<\infty .
$$

Example 4.2. Consider $M=[0,1]$ and the operator $L=-i \frac{d}{d x}$ on $M^{\circ}=(0,1)$ with the domain

$$
D(L)=\left\{f \in W_{2}^{1}[0,1] \mid a f(0)+b f(1)+\int_{0}^{1} f(x) q(x) d x=0\right\},
$$

where $a \neq 0, b \neq 0$, and $q \in C^{1}[0,1]$, here $W_{2}^{1}[0,1]:=\left\{f \in L^{2}[0,1] \mid f^{\prime} \in L^{2}[0,1]\right\}$. Under the assumption that $a+b+\int_{0}^{1} q(x) d x=1$, we have the inverse $L^{-1}$ exists and is bounded from $L^{2}[0,1]$ to $D(L)$. The operator $L$ has a discrete spectrum which can be enumerated by $\lambda_{j}=2 \pi j-i \ln (-a / b)+\alpha_{j}$, for $j \in \mathbb{Z}$, where the sequence $\alpha_{j}$ satisfies that for any $\epsilon>0, \sum_{j \in \mathbb{Z}}\left|\alpha_{j}\right|^{1+\epsilon}<\infty$. If $m_{j}$ denotes the multiplicity of the eigenvalue $\lambda_{j}$, then $m_{j}=1$ for sufficiently large $|j|$, and the system of eigenfunctions are given by

$$
u_{j k}=\frac{(i x)^{k}}{k !} e^{i \lambda_{j} x}, \text { for } 0 \leq k \leq m_{j}-1 \text { and } j \in \mathbb{Z} .
$$

Let $j_{0} \in \mathbb{N}$ large enough so that $m_{j}=1$ for $|j| \geq j_{0}$. The global symbol $\sigma_{L, L}(x, j)$ of $L$ with respect to $L$ is given by

$$
\sigma_{L, L}(x, j)=\left\{\begin{array}{cc}
\frac{x^{2 k}}{k ! l^{2}} \lambda_{j}, & x \in[0,1] ; 0 \leq k \leq m_{j}-1,|j| \leq j_{o} \\
\lambda_{j}, & |j| \geq j_{0}
\end{array}\right.
$$

for some $j_{0} \in \mathbb{N}$. It follows from the functional calculus that $L^{-1}$ has a discrete spectrum given by $\lambda_{j}^{-1}$ with $j \in \mathbb{Z}$. Since $\lambda_{j} \mid=O(j)$ for $j \in \mathbb{Z}$, we have $\sum_{|j| \leq N}\left|\lambda_{j}^{-1}\right|=O(\ln (2 N+1))$. Therefore, $L^{-1}$ is a pseudo-differential operator which lies in $\mathcal{L}^{(1, \infty)}\left(L^{2}[0,1]\right)$ with symbol $\sigma_{L^{-1}, L}(\xi)=\left(\sigma_{L, L}(\xi)\right)^{-1},|\xi| \geq j_{0}$, and

$$
\begin{aligned}
\operatorname{Tr}_{\omega}\left(L^{-1}\right) & =\lim _{N \rightarrow \infty} \frac{1}{\ln (2 N+1)} \sum_{|j| \leq N}\left|\lambda_{j}^{-1}\right| \\
& =\lim _{N \rightarrow \infty} \frac{1}{\ln (2 N+1)} \sum_{j_{0} \leq|j| \leq N}\left|\left(\sigma_{L, L}(x, j)\right)^{-1}\right| \\
& =\lim _{N \rightarrow \infty} \frac{1}{\ln (2 N+1)} \sum_{|j| \leq N} \frac{2 \pi j+i \ln (-a / b)+\overline{\alpha_{j}}}{\left(2 \pi j+\operatorname{Re}\left(\alpha_{j}\right)\right)^{2}+\left(\operatorname{Im}\left(\alpha_{j}\right)+\ln (b / a)\right)^{2}} .
\end{aligned}
$$

Example 4.3. Let us assume that $A$ is a left-invariant operator on $\mathrm{SU}(2) \cong \mathbb{S}^{3}$. We recall that the unitary dual of $\mathrm{SU}(2)$ (see [31]) can be identified as

$$
\widehat{\mathrm{SU}}(2) \equiv\left\{\left[t_{l}\right]: 2 l \in \mathbb{N}, d_{l}:=\operatorname{dim} t_{l}=(2 l+1)\right\} .
$$

There are explicit formulae for $t_{l}$ as function of the Euler angles in terms of the so-called Legendre-Jacobi polynomials, see [31]. In this case, if A is Dixmier traceable with symbol 
The Dixmier trace and the Wodzicki residue for global pseudo-differential operators

$\sigma\left(\left[t_{l}\right]\right) \equiv \sigma(l)$, then

$$
\begin{aligned}
\operatorname{Tr}_{w}(A) & =\lim _{N \rightarrow \infty} \frac{1}{\log \left(\sum_{l \leq \frac{N}{2}, l \in \frac{1}{2} \mathbb{N}_{0}} d_{l}^{2}\right)} \sum_{l \leq \frac{N}{2}, l \in \frac{1}{2} \mathbb{N}_{0}} d_{\left[t_{l}\right]} \operatorname{Tr}[|\sigma(l)|] \\
& =\lim _{N \rightarrow \infty} \frac{1}{\log \left(\sum_{l \leq \frac{N}{2}, l \in \frac{1}{2} \mathbb{N}_{0}}(2 l+1)^{2}\right)} \sum_{l \leq \frac{N}{2}, l \in \frac{1}{2} \mathbb{N}_{0}}(2 l+1) \operatorname{Tr}[|\sigma(l)|] \\
& =\lim _{N \rightarrow \infty} \frac{1}{\log \left[\frac{1}{6}(N+1)\left(2 N^{2}+7 N+1\right)\right]} \sum_{l \leq \frac{N}{2}, l \in \frac{1}{2} \mathbb{N}_{0}}(2 l+1) \operatorname{Tr}[|\sigma(l)|] .
\end{aligned}
$$

For example, if $A=\left(I-\mathcal{L}_{\mathrm{SU}(2)}\right)^{-\frac{\varkappa}{2}}$, is the Bessel potential of order $-\varkappa=-\operatorname{dim} \mathrm{SU}(2)=$ -3 , then $A$ is Dixmier traceable, $\sigma(l)=(1+l(l+1))^{-\frac{3}{2}}$ and

$$
\begin{aligned}
& \operatorname{Tr}_{w}\left(\left(I-\mathcal{L}_{\mathrm{SU}(2)}\right)^{-\frac{3}{2}}\right) \\
& =\lim _{N \rightarrow \infty} \frac{1}{\log \left[\frac{1}{6}(N+1)\left(2 N^{2}+7 N+1\right)\right]} \sum_{l \leq \frac{N}{2}, l \in \frac{1}{2} \mathbb{N}_{0}}(2 l+1)^{2}[1+l(l+1)]^{-\frac{3}{2}} \\
& =\lim _{N \rightarrow \infty} \frac{1}{\log \left[\frac{1}{6}(N+1)\left(2 N^{2}+7 N+1\right)\right]} \sum_{n=0}^{N}(n+1)^{2}\left[1+\frac{n}{2}\left(\frac{n}{2}+1\right)\right]^{-\frac{3}{2}} \\
& =\lim _{N \rightarrow \infty} \frac{1}{\log \left[\frac{1}{6}(N+1)\left(2 N^{2}+7 N+1\right)\right]} \sum_{n=1}^{N+1} \frac{n^{2}}{8}\left[n^{2}+3\right]^{-\frac{3}{2}} \\
& \sim 0.03935 \ldots \text { (numerical evidence). }
\end{aligned}
$$

A similar result can be obtained if we consider the operator $\left(I-\mathcal{L}_{\text {sub }}\right)^{-\frac{3}{2}}$, where $\mathcal{L}_{\text {sub }}:=$ $D_{1}^{2}+D_{2}^{2}$ is the sub-Laplacian on $\mathrm{SO}(3)$; here $D_{1}$ and $D_{2}$ are the derivatives in the corresponding variables to the Euler angles for $\mathrm{SO}(3)$ (see [31, 32]).

Example 4.4. Let $A$ be a left-invariant pseudo-differential operator Dixmier traceable on $\mathrm{SU}(3)$. The Lie group $\mathrm{SU}(3)$ has dimension 8 and 3 positive square roots $\alpha, \beta$ and $\rho$ with the property $\rho=\frac{1}{2}(\alpha+\beta+\rho)$. We define the weights

$$
\sigma=\frac{2}{2} \alpha+\frac{1}{3} \beta, \tau=\frac{1}{3} \alpha+\frac{2}{3} \beta
$$

With the notations above, the unitary dual of $\mathrm{SU}(3)$ can be identified with

$$
\widehat{\mathrm{SU}}(3) \equiv\left\{\lambda:=\lambda(a, b)=a \sigma+b \tau: a, b \in \mathbb{N}_{0}, d_{\lambda}=\frac{1}{2}(a+1)(b+1)(a+b+2)\right\} .
$$

The Dixmier trace of $A$, once denoted its full symbol by $\sigma(\lambda) \equiv \sigma(\lambda(a, b))$, is given by the 
expression

$$
\begin{aligned}
& \operatorname{Tr}_{w}(A) \\
& =\lim _{N \rightarrow \infty} \frac{1}{\log \left[\sum_{0 \leq a+b \leq N} d_{\lambda(a, b)}\right]} \times \sum_{0 \leq a+b \leq N} \frac{1}{2}(a+1)(b+1)(a+b+2) \operatorname{Tr}[|\sigma(\lambda(a, b))|] \\
& =\lim _{N \rightarrow \infty} \frac{1}{\log \left[\frac{1}{120}(N+1)(N+2)(N+3)(N+4)(2 N+5)\right]} \times \\
& \sum_{(a, b): 0 \leq a+b \leq N} \frac{1}{2}(a+1)(b+1)(a+b+2) \operatorname{Tr}[|\sigma(\lambda(a, b))|] .
\end{aligned}
$$

Since, the eigenvalues of the Laplacian $\mathcal{L}_{\mathrm{SU}(3)}$ on $S U(3)$ are of the form

$$
-c(\lambda)=-\frac{1}{9}\left(a^{2}+b^{2}+a b+3 a+3 b\right)
$$

the symbol of the operator $A=\left(I-\mathcal{L}_{\mathrm{SU}(3)}\right)^{-\frac{8}{2}}$ (which is Dixmier traceable) is given by

$$
\sigma(\lambda)=(1+c(\lambda))^{-4}
$$

Hence,

$$
\begin{aligned}
& \operatorname{Tr}_{w}\left(\left(I-\mathcal{L}_{\mathrm{SU}(3)}\right)^{-\frac{8}{2}}\right) \\
& =\lim _{N \rightarrow \infty} \frac{1}{\log \left[\frac{1}{120}(N+1)(N+2)(N+3)(N+4)(2 N+5)\right]} \times \\
& \quad \sum_{(a, b): 0 \leq a+b \leq N} \frac{1}{36}(a+1)^{2}(b+1)^{2}(a+b+2)^{2}\left[1+\left(a^{2}+b^{2}+a b+3 a+3 b\right)\right]^{-4} .
\end{aligned}
$$

Remark 4.5. Similar examples to the considered above can be obtained if we take, for example, every $n$-torus $\mathbb{T}^{n} \cong \mathbb{R}^{n} / \mathbb{Z}^{n}$ or the compact Lie group $\mathrm{SO}(3) \cong \mathbb{R} \mathbb{P}^{3}$, spheres $\mathbb{S}^{n}$ and arbitrary real and complex projective spaces.

Example 4.6. Let us assume that $G$ is a compact Lie group of dimension $\varkappa$, and let us define $A:=a(x)\left(-\mathcal{L}_{G}\right)^{-\frac{\varkappa}{2}}$. We consider $a(x)$ positive and smooth in order that $A$ will be a positive operator. The global symbol of $A$ is given by

$$
\sigma_{A}(x, \xi):=a(x) \lambda_{[\xi]}^{-\frac{\varkappa}{2}} I_{d_{\xi}}, \quad \lambda_{[\xi]} \neq 0,
$$

and by using (11) we have that

$$
\operatorname{res}(A)=\frac{1}{\operatorname{dim}(G)} \int_{G} a(x) d x \times \lim _{N \rightarrow \infty} \frac{1}{\log N} \sum_{\xi:\langle\xi\rangle \leq N} d_{\xi}^{2} \lambda_{[\xi]}^{-\frac{\varkappa}{2}} .
$$

In particular, for $G=\mathbb{T}^{\varkappa}$, where $\mathbb{T} \equiv[0,1), d_{\xi}=1$, and $\lambda_{[\xi]}=1 / 4 \pi^{2}|\xi|^{2}, \xi \in \mathbb{Z}$. In this 
case,

$$
\begin{aligned}
& \lim _{N \rightarrow \infty} \frac{1}{\log N} \sum_{\xi:\langle\xi\rangle \leq N} d_{\xi}^{2} \lambda_{[\xi]}^{-\frac{\varkappa}{2}} \\
& \asymp \lim _{N \rightarrow \infty} \frac{1}{\log N} \sum_{\xi \in \mathbb{Z}^{\varkappa}, 1 \leq|\xi| \leq N}|\xi|^{-\varkappa} \asymp \lim _{N \rightarrow \infty} \frac{1}{\log N} \int_{1 \leq|\xi| \leq N}|\xi|^{-\varkappa} d \xi \\
& \asymp \lim _{N \rightarrow \infty} \frac{1}{\log N} \log (N)=1 .
\end{aligned}
$$

Consequently,

$$
\operatorname{res}(A) \asymp \frac{1}{\varkappa} \int_{\mathbb{T}^{\varkappa}} a\left(x_{1}, \cdots, x_{\varkappa}\right) d x_{1} \cdots d x_{\varkappa},
$$

which is a well known fact (see e.g. Connes [13]).

Acknowledgements. The authors thank Vishvesh Kumar for useful discussions on the subject.

\section{References}

[1] Barraza E.S. and Cardona D., "On nuclear Lp multipliers associated to the harmonic oscillator", in Analysis and Partial Differential Equations: Perspectives from Developing Countries. Springer Proceedings in Mathematics \& Statistics vol 275 (eds Ruzhansky M. and Delgado J.) Springer, Cham (2019), 31-41.

[2] Boutet de Monvel L., "Boundary problems for pseudo-differential operators", Acta Math. 126 (1971), No. 1-2, 11-51.

[3] Chatzakou M., Delgado J.m and Ruzhansky M., "On a class of anharmonic oscillators", arXiv:1811.12566.

[4] Cardona D., "Nuclear pseudo-differential operators in Besov spaces on compact Lie groups", J. Fourier Anal. Appl. 23 (2017), No. 5, 1238-1262.

[5] Cardona D., "A brief description of operators associated to the quantum harmonic oscillator on Schatten-von Neumann classes", Rev. Integr. temas mat. 36 (2018), No. 1, 49-57.

[6] Cardona D., "On the nuclear trace of Fourier Integral Operators", Rev. Integr. temas mat. 37 (2019), No. 2, 219-249.

[7] Cardona D., Del Corral C. and Kumar V., "Dixmier traces for discrete pseudo-differential operators", arXiv:1911.03924.

[8] Cardona D. and Kumar V., "Multilinear analysis for discrete and periodic pseudodifferential operators in Lp spaces", Rev. Integr. temas mat. 36 (2018), No. 2, 151-164.

[9] Cardona D. and Kumar V., " $L^{p}$-boundedness and $L^{p}$-nuclearity of multilinear pseudodifferential operators on $Z^{n}$ and the torus $T^{n}$, J. Fourier Anal. Appl. 25 (2019), No. 6, 2973-3017.

[10] Cardona D. and Kumar V., "The nuclear trace of vector-valued pseudo-differential operators with applications to Index theory", arXiv:1901.10010.

Vol. 38, $\left.\mathrm{N}^{\circ} 1,2020\right]$ 
[11] Cardona D. and Barraza E.S., "Characterization of nuclear pseudo-multipliers associated to the harmonic oscillator", Politehn. Univ. Bucharest Sci. Bull. Ser. A Appl. Math. Phys 80 (2018), No. 4, 163-172.

[12] Cardona D. and Del Corral C., "The Dixmier trace and the noncommutative residue for multipliers on compact manifolds", arXiv:1703.07453.

[13] Connes A., Noncommutative geometry, Academic Press Inc., San Diego, 1994.

[14] Delgado J. and Ruzhansky M., "Schatten-von Neumann classes of integral operators", arXiv:1709.06446.

[15] Delgado J. and Ruzhansky M., "Fourier multipliers in Hilbert spaces", arXiv:1707.03062.

[16] Delgado J. and Ruzhansky M., " $L^{p}$-nuclearity, traces, and Grothendieck-Lidskii formula on compact Lie groups", J. Math. Pures Appl. 102 (2014), No. 1, 153-172.

[17] Delgado J. and Ruzhansky M., "Schatten classes on compact manifolds: Kernel conditions", J. Funct. Anal. 267 (2014), No. 3, 772-798.

[18] Delgado J. and Ruzhansky M., "Kernel and symbol criteria for Schatten classes and rnuclearity on compact manifolds", C. R. Acad. Sci. Paris. 352 (2014), No. 10, 779-784.

[19] Delgado J. and Ruzhansky M., " $L^{p}$-bounds for pseudo-differential operators on compact Lie groups", arXiv:1605.07027.

[20] Delgado J. and Ruzhansky M., "Fourier multipliers, symbols and nuclearity on compact manifolds", arXiv:1404.6479.

[21] Delgado J., Ruzhansky M. and Tokmagambetov N., "Schatten classes, nuclearity and nonharmonic analysis on compact manifolds with boundary", arXiv:1505.02261.

[22] Dixmier J., "Existence de traces non normales", C. R. Acad. Sci. Paris. Sér A-B 262 (1966), 1107-1108.

[23] Fedosov B., Golse F., Leichtnam E. and Schrohe E., "The Noncommutative Residue for Manifolds with Boundary", J. Funct. Anal. 142 (1996), No. 1, 1-31.

[24] Ghaemi M. B., Jamalpourbirgani M. and Wong M. W., "Characterizations, adjoints and products of nuclear pseudo-differential operators on compact and Hausdorff groups", Politehn. Univ. Bucharest Sci. Bull. Ser. A Appl. Math. Phys. 79 (2017), No. 4, 207-220.

[25] Grubb G. and Schrohe E., "Trace expansions and the noncommutative residue for manifolds with boundary", J. Reine Angew. Math. 536 (2001), 167-207.

[26] Hörmander L., The Analysis of the linear partial differential operators III, Springer-Verlag, Berlin, 1985.

[27] Kumar V.n and Wong M. W., " $C^{*}$-algebras, $H^{*}$-algebras and trace ideals of pseudodifferential operators on locally compact, Hausdorff and abelian groups", J. Pseudo-Differ. Oper. Appl. 10 (2019), No. 2, 269-283.

[28] Nest R. and Schrohe E., "Dixmier's trace for boundary value problems", Manuscripta math. Springer-Verlag 96 (1998), No. 2, 203-218.

[29] Pietsch A. "Traces and residues of pseudo-differential operators on the torus", Integr. Equ. Oper. Theory. 83 (2015), No. 1, 1-23. 
[30] Ruzhansky M. and Tokmagambetov N., "Nonharmonic Analysis of Boundary Value Problems“, International Mathematics Research Notices, (2016), No. 12, 3548-3615.

[31] Ruzhansky M., Turunen V., "Pseudo-Differential Operators and Symmetries.", Background Analysis and Advanced Topics, Birkhäuser-Verlag, Basel, 2010.

[32] Ruzhansky M., Wirth, J., " $L^{p}$ Fourier multipliers on compact Lie groups.", Math. Z., 280 (2015), No. 3-4, 621-642.

[33] Schrohe, E., "Noncommutative Residues, Dixmier's Trace, and Heat Trace Expansions on Manifolds with Boundary.", Amer. Math. Soc. Providence, Providence, RI, No. 161-186, 1999.

[34] Wodzicki M., "Noncommutative residue. I. Fundamentals.", Yu. I. Manin (ed.), Lecture Notes in Math., 1289, Springer, Berlin, 1987. 